\title{
Maximal Homomorphism in Fundamental Theorem of Homomorphism Semiring
}

\author{
Riski Ryan Hardiansyah*, Khurul Wardati**, Muhamad Zaki Riyanto*** \\ Mathematic Department, Faculty of Science and Technology, UIN Sunan Kalijaga Yogyakarta \\ Jl. Marsda Adisucipto No 1 Yogyakarta 55281, Indonesia. Tel. +62-274-540971, Fax. +62-274-519739. \\ Email: riskiryanhardiansyah@gmail.com*, khurulwardati@gmail.com**, zakimath@gmail.com***
}

\begin{abstract}
Hardiansyah R R, Wardati K, Riyanto M Z. 2017. Maximal Homomorphism in Fundamental Theorem of Homomorphism Semiring. Proc Internat Conf Sci Engin 1: 189-192. Semiring is a generalization of ring. Some concepts in ring can be developed in semiring. The contructions of quotient semiring requires a specific ideal, namely $Q$-ideal. Different to the ring theory, the fundamental theorems of homomorphism on semiring needs a concept of maximal homomorphism, science the kernel of any maximal homomorphism is always $Q$-ideal.
\end{abstract}

Keywords: Semiring, Quotient Semiring, Maximal Homomorphism, Fundamental Theorem Homomorphism of Semiring

\section{INTRODUCTION}

Ring is one of algebraic structures. The ring structure can be generalized to be semiring. Its generalization is the replacement of commutative additive group into commutative additive monoid ([1] [2] [3]). It needs to examine some of the properties of this generalization.

Different to the ring theory, the construction of a quotient semiring requires a more specific ideal called Q-ideal [2]. For every ideal in a ring can be constructed a quotient ring, but not for any ideal in a semiring. This will be given by a counter example, an ideal in a semiring that does not form a quotient semiring. In addition, it will be further discussed the construction of a quotient semiring.

The fundamental theorem of homomorphism in semiring has been studied in [1]. For any kernel of homomorphism in either a ring or a semiring is an ideal. However, the kernel in semiring does not necessarily form a quotient semiring. This statement requires a counter example. This article will discuss a maximal homomorphism whose kernel is always Q-ideal. Maximum homomorphism is instrumental in the construction of the fundamental theorem of homomorphism semiring. The Maximal homomorphism plays an important role in the construction of the fundamental theorem of homomorphism in semiring.

\section{QUOTIENT SEMIRINGS}

To discuss quotient semirings, we need the definition of semiring and its ideal. Semiring is a generalization of ring.

Definition 1. [1] A set $R$ with two binary operations named addition and multiplication will be called a semiring provided

a. $R$ over addition is commutative monoid b. $R$ over multiplication is semigrup

c. There exist $0_{R} \in R$ such that $a 0_{R}=0_{R} a=0_{R}$ for each $a \in R$

d. Multiplication distributes over addition both from the left and the right.

As the quotient ring, a quotient semiring requires an ideal of semiring. The difference between ideal in a ring and ideal in semiring is on the first axiom.

Definition 2. [2] A subset $I$ of semiring $R$ is called an ideal, if for each $a, b \in I$ and $r \in R$ implies $a+b \in I, r a \in I$ and $a r \in I$.

Example 3. The set of nonnegative integers is denoted by $\mathbb{Z}_{\text {₹0 }}$.

1. The set $\mathbb{Z}_{2_{0}}$ is a semiring over addition and multiplication with the zero element 0 . It is clear that $2 \mathbb{Z}_{20}$ is an ideal of $\mathbb{Z}_{20}$.

2. The set $R=\mathbb{Z}_{20} \cup\{\infty\}$ is a semiring with the operations defined by $a \oplus b=\max (a, b)$ and $a \odot b=\min (a, b)$ and the zero element is $\infty$. Let $I=\{a \in R \mid a \leq 4\}$ then $I$ is an ideal of $R$.

In the ring theory, a quotient ring is a partition of a ring formed by an ideal of the ring. If $I$ be an ideal of a ring $R$, then we can form the quotient ring, $R / I=\{r+I \mid r \in R\}$, which is a partition of $R$. However, if $R$ is a semiring, then the set $R / I=\{r+I \mid r \in R\}$ is not necessarily a partition of $R$.

Let $I=\{a \in R \mid a \leq 4\}$ be an ideal of $R=\mathbb{Z}_{20} \cup\{\infty\}$ in Example 3. et $1,2 \in I$ then we have $1 \oplus I=\{1,2,3,4\} \quad \neq \quad 2 \oplus I=\{2,3,4\} \quad$ and $(1 \oplus I) \cap(3 \oplus I) \neq \phi$. Thus $R / I=\{r+I \mid r \in R\}$ is not a partition of $R$. It is shown that if $I$ is an ideal of 
semiring $R$, then $R / I$ is not necessarily a quotient semiring. The problem is how to construct a set of cosets of an ideal $I$ in the semiring $R$ that forms a partition of $R$. The following is given a special ideal in the semiring called ideal partition or Q-ideal.

Definition 4. [2] An ideal $I$ of a semiring $R$ is called a partitioning ideal or $Q$-ideal if there is a non-empty subset $Q$ of $R$ such that:

a. $R=\bigcup_{q \in Q}\{q+I \mid q \in Q\}$.

b. If $q_{1}, q_{2} \in Q$ then $\left(q_{1}+I\right) \cap\left(q_{2}+I\right) \neq \phi$ if and only if $q_{1}=q_{2}$.

Example 5. The set of all non-negative even integers $2 \mathbb{Z}_{20}$ is an ideal of the semiring $\mathbb{Z}_{20}$. Furthermore, $2 \mathbb{Z}_{20}$ is a $Q$-ideal of the semiring $\mathbb{Z}_{20}$ with $Q=\{0,1\}$.

Based on Definition 4., the partition of $R$ is very dependent on the $Q$-ideal for a subset $Q$ of $R$. If $I$ is a $Q$ ideal of $R$ then $R / I=\{q+I \mid q \in Q\}$ is a partition of $R$. Here is given Lemma to prove $R / I$ is a quotient semiring.

Lemma 6. [1] Let $I$ be a $Q$-ideal in a semiring $R$. If $x \in R$, then there exists a unique $q \in Q$ such that $(x+I) \subseteq(q+I)$.

The above lemma states that an element in semiring $R$ can not be in two different classes. We will show that any $Q$-ideal $I$ in the semiring forms a quotient semiring.

Theorem 7. [1] Let $I$ be a $Q$-ideal in the semiring $R$, then $R / I=\{q+I \mid q \in Q\}$ is a semiring called a quotient semiring over binary operations $\oplus$ and $\odot$ defined as

$$
\begin{aligned}
& \left(q_{1}+I\right) \oplus\left(q_{2}+I\right)=\left(q_{3}+I\right) \\
& \left(q_{1}+I\right) \odot\left(q_{2}+I\right)=\left(q_{4}+I\right) .
\end{aligned}
$$

Where $q_{3}, q_{4} \in Q$ are the unique elements in $Q$ such that $q_{1}+q_{2}+I \subseteq q_{3}+I, q_{1} q_{2}+I \subseteq q_{4}+I$.

Proof. It is easy to show that $\oplus$ and $\odot$ are associative, $\oplus$ is commutative, and $\odot$ is left and right distributive over $\oplus$. We will show that $R / I$ has a zero element over $\oplus$. Let $I$ be a ideal $Q$-ideal in $R$, then based on Lemma 6. there exists a unique $q_{0} \in Q$ such that $\left(0_{R}+I\right) \subset\left(q_{0}+I\right) . \quad$ Note that $0_{R} \in q_{0}+I$. Therefore, there exists $k \in I$ such that $0_{R}=q_{0}+k$. It is claimed that $q_{0}+I$ is the zero element in $(R / I, \oplus)$. We will prove that for every $A \in R / I$ with $A=q_{1}+I$ then $A \oplus q_{0}+I=A$. Let $A \in R / I$ with $A=q_{1}+I$, it is found that $A \oplus q_{0}+I=$ $\left(q_{1}+I\right) \oplus\left(q_{0}+I\right)=\left(q_{2}+I\right)$, where $q_{2}$ is a unique element in $Q$ such that $\left(\left(q_{1}+q_{0}\right)+I\right) \subseteq\left(q_{2}+I\right)$.
Since $\quad k \in I, \quad$ we get $\left(\left(q_{1}+q_{0}\right)+k\right) \in\left(\left(q_{1}+q_{0}\right)+I\right) \subseteq\left(q_{2}+I\right)$. Since $0_{R}=q_{0}+k$, we get $q_{1}=q_{1}+q_{0}+k \in q_{2}+I$. On the other hand, $q_{1} \in q_{1}+I$. It implies that $q_{1}+I \cap q_{2}+I \neq \emptyset$. Since $I$ is a $Q$-ideal and $q_{1}+I \cap q_{2}+I \neq \emptyset$., we get $q_{1}=q_{2}$. Thus, $q_{0}+I$ is the zero element in $(R / I, \oplus)$, i.e $\left(q_{1}+I\right) \oplus\left(q_{0}+I\right)=\left(q_{0}+I\right) \oplus\left(q_{1}+I\right)=\left(q_{1}+I\right)$.

\section{MAXIMAL HOMOMORPHISM}

A homomorphism of semiring has the same definition as the homomorphism of ring. Homomorphism is a function that preserves the operations of addition and multiplication.

Definition 8.[1] Let $R$ and $R^{\prime}$ be semirings and $f: R \rightarrow R^{\prime}$ be a function. The function $f$ is called homomorphism of semiring if for every $a, b \in R$ such that $\quad f(a+b)=f(a)+f(b) \quad$ and $f(a b)=f(a) f(b)$. We define the kernel of homomorphism $f$ as the set $\operatorname{ker}(f)=\left\{a \in R \mid f(a)=0^{\prime}\right\}$.

Example 9. Given semiring $R=\mathbb{Z}_{20} \cup\{\infty\}$ in Example 3 and subsemiring $R^{\prime}=\{0,1\}$ of $R$. Defined function $f$ i.e. $f: R \rightarrow R^{\prime}$, with $f(a)=0$ if $a \leq 4$ and $f(a)=1$ if $a>4$ for each $a \in R$. It is clear that $f$ is a homomorphism of semiring.

Consider Example 9, that $\operatorname{ker}(f)=\{a \in R \| a \leq 4\}$ is an ideal of semiring $R$. However, ${ }^{R} / \operatorname{ker}(f)$ is not a partition as described above. The kernel of any homomorphism of semiring would be the $Q$-ideal if the homomorphism is a maximal homomorphism.

Definition 10. [1] A homomorphism $f$ from a semiring $R$ onto a semirirng $R^{\prime}$ is said to be maximal if for each $x \in R^{\prime}$ there exists $c_{x} \in f^{-1}(\{x\})$ such that $a+\operatorname{ker}(f) \subset c_{x}+\operatorname{ker}(f)$ for each $a \in f^{-1}(\{x\})$.

Example 11. Given the semiring $\left(\mathbb{Z}_{20},{ }, \cdot\right)$ and the quotient semiring $\left(\mathbb{Z}_{20} / 2 \mathbb{Z}_{2_{0}}, \oplus . \odot\right)$ where $2 \mathbb{Z}_{20}$ is a Q-ideal with $Q=\{0,1\}$. We define a function $f: \mathbb{Z}_{20} / 2 \mathbb{Z}_{\mathrm{z} 0} \rightarrow \mathbb{Z}_{\mathrm{z} 0}$ by $f(a)=q+2 \mathbb{Z}_{\mathrm{zo}}$ for each $a \in \mathbb{Z}_{20}$ with $a+2 \mathbb{Z}_{20} \subset q+2 \mathbb{Z}_{20}$. It is easy to show that $f$ is a maximal homomorphism with $\operatorname{ker}(f)=2 \mathbb{Z}_{20}$.

Example 12. The homomorphism in Example 9 is not a maximal homomorphism, because $a+\operatorname{ker}(f)=\{a\}$, 
for every $a \in f^{-1}(\{1\})$, there is not exist $c_{1} \in f^{-1}(\{1\})$ such that $a+\operatorname{ker}(f) \subset c_{1}+\operatorname{ker}(f)$.

The concept of maximal homomorphism will be used to construct the fundamental theorem of homomorphism in semiring. The following lemmas are some properties of the maximal homomorphism.

Lemma 12.[3] Let $f$ be a homomorphism of the semiring $R$ onto the semiring $R^{\prime}$. If $f$ is the maximal, then $\operatorname{ker}(f) \quad$ is $Q$-ideal with $Q=\left\{c_{a} \mid c_{a} \in f^{-1}(\{1\}), a \in R^{\prime}\right\}$.

Lemma 13.[3] Let $R, R^{\prime}, f$ and $Q$ be as stated in Lemma 12 , and let $c_{a}, c_{b}, c_{c} \in Q$ such that:

$$
\begin{aligned}
& \text { a. If } c_{a}+c_{b}+\operatorname{ker}(f) \subset c_{c}+\operatorname{ker}(f) \text {, then } \\
& a+b=c \text {. }
\end{aligned}
$$$$
\text { b. If } c_{a} c_{b}+\operatorname{ker}(f) \subset c_{c}+\operatorname{ker}(f) \text {, then } a b=c \text {. }
$$

Lemma 12 and 13, play an important role to prove the fundamental theorem of homomorphism in semiring. The epimorphism and the maximal are principal conditions of the fundamental theorem of homomorphism in semiring.

Theorem 14 [3]. If $f$ is a maximal homomorphism from the semiring $R$ onto the semiring $R^{\prime}$, then $R / \operatorname{ker}(f) \cong R^{\prime}$.

$$
\text { Proof. Define } \quad \bar{f}: R / \operatorname{ker}(f) \rightarrow R^{r} \quad \text { by }
$$

$\bar{f}\left(c_{a}+\operatorname{ker}(f)\right)=a$ for each $c_{a} \in Q$. It will be shown $\bar{f}$ is an isomorphism.

\section{It will be shown that $\bar{f}$ is a function.}

Let $\left(c_{a}+\operatorname{ker}(f)\right),\left(c_{b}+\operatorname{ker}(f)\right) \in \in^{R} / \operatorname{Ker}(f)$ with $c_{a}+\operatorname{ker}(f)=c_{b}+\operatorname{ker}(f)$

It's means that $c_{a}+\operatorname{ker}(f) \cap c_{b}+\operatorname{ker}(f) \neq \emptyset$. Using Lemma $13, \operatorname{ker}(f)$ is a $Q$-ideal such that $c_{a}=c_{b}$. Since $c_{a} \in f^{-1}(\{a\})$ and $c_{b} \in f^{-1}(\{a\})$ then $f\left(c_{a}\right)=a$ and $f\left(c_{b}\right)=b$, such that

$$
c_{a}=c_{b} \Leftrightarrow f\left(c_{a}\right)=f\left(c_{b}\right) \Leftrightarrow a=b
$$

We obtain

$\bar{f}\left(c_{a}+\operatorname{Ker}(f)\right)=a=b=\bar{f}\left(c_{b}+\operatorname{Ker}(f)\right)=b$.

It is proven that $\bar{f}$ is a function.

\section{It will be shown that $\bar{f}$ is a homomorphism.}

Let $\quad\left(c_{a}+\operatorname{ker}(f)\right),\left(c_{b}+\operatorname{ker}(f)\right) \in^{R} / \operatorname{Ker}(f)$.

Assume that

$\left(c_{a}+\operatorname{ker}(f)\right) \oplus\left(c_{b}+\operatorname{ker}(f)\right)=c_{c}+\operatorname{ker}(f)$

and

$\left(c_{a}+\operatorname{ker}(f)\right) \odot\left(c_{b}+\operatorname{ker}(f)\right)=c_{a}+\operatorname{ker}(f)$

Where $c_{c}, c_{d}$ are the unique elements in $Q$ such that $c_{a}+c_{b}+\operatorname{ker}(f) \subset c_{c}+\operatorname{ker}(f)$

and $c_{a} c_{b}+\operatorname{ker}(f) \subset c_{a}+\operatorname{ker}(f)$. By using Lemma 14 then

$\bar{f}\left(\left(c_{a}+\operatorname{ker}(f)\right) \oplus\left(c_{b}+\operatorname{ker}(f)\right)\right)=\bar{f}\left(c_{c}+\operatorname{ker}(f)\right)$

$=c=a+b$

$=\bar{f}\left(c_{a}+\operatorname{ker}(f)\right)+\bar{f}\left(c_{b}+\operatorname{ker}(f)\right)$

and

$\bar{f}\left(\left(c_{a}+\operatorname{ker}(f)\right) \odot\left(c_{b}+\operatorname{ker}(f)\right)\right)=\bar{f}\left(c_{a}+\operatorname{ker}(f)\right)$

$=d=a b$

$=\bar{f}\left(c_{a}+\operatorname{ker}(f)\right) \bar{f}\left(c_{b}+\operatorname{ker}(f)\right)$.

Hence, $\bar{f}$ is a homomorphism.

3. It will be shown that $\bar{f}$ is injective.

Let $\left(c_{a}+\operatorname{ker}(f)\right)_{0}\left(c_{b}+\operatorname{ker}(f)\right) \in \mathbb{R}^{R} / \operatorname{ker}(f)$ with

$\bar{f}\left(c_{a}+\operatorname{ker}(f)\right)=\bar{f}\left(c_{b}+\operatorname{ker}(f)\right)$

It's mean $a=b$. Since $c_{a} \in f^{-1}(\{a\})$ then for every $x \in f^{-1}(\{a\})$, we have

$x+\operatorname{ker}(f) \subset c_{a}+\operatorname{ker}(f)$

And since $c_{b} \in f^{-1}(\{b\})$ then for every $x \in f^{-1}(\{b\})$, we have

$x+\operatorname{ker}(f) \subset c_{b}+\operatorname{ker}(f)$

Then, since $a=b$, such that

$f^{-1}(\{a\})=\{x \in R \mid f(x)=a\}$

$=\{x \in R \mid f(x)=b\}$

$=f^{-1}(\{b\})$

So that it is obtained that for every $x \in f^{-1}(\{a\})=f^{-1}(\{b\})$ implies that

$x+\operatorname{ker}(f) \subset c_{a}+\operatorname{ker}(f)$

and

$x+\operatorname{ker}(f) \subset c_{b}+\operatorname{ker}(f)$

Using Lemma 12, we have $\operatorname{ker}(f)$ is $Q$-ideal. Since $c_{a}+\operatorname{ker}(f) \cap c_{b}+\operatorname{ker}(f) \neq \emptyset$, then $c_{a}=c_{b}$ such that $c_{a}+\operatorname{ker}(f)=c_{b}+\operatorname{ker}(f)$.

Hence, $\bar{f}$ is injective.

\section{It will be shown that $\bar{f}$ is a surjective function.}

Let $a \in R^{\prime}$. Since $f$ is a maximal homomorphism, using Definition 10., that for every $a \in R^{\prime}$. there exists $c_{a} \in f^{-1}(\{a\})$ implies that for every $x \in f^{-1}(\{a\})$ we have $x+\operatorname{ker}(f) \subseteq c_{a}+\operatorname{ker}(f)$. Consequently, for every $a \in R^{\prime}$ there is $c_{a}+\operatorname{ker}(f)$ such that $a=\bar{f}\left(c_{a}+\operatorname{ker}(f)\right)$

Hence, $\bar{f}$ is a surjective function.

Therefore, $\bar{f}$ is an isomorphism. In other words, $R / \operatorname{ker}(f) \cong R^{\prime}$.

\section{CONCLUSIONS}

The ring structure can be generalized into semiring. One of the properties of semiring is that not all ideal in semiring can form a quotient semiring. To form a quotient semiring requires a special ideal named Q- 
ideal. The construction of the fundamental theorem of homomorphism in semiring uses the concept of the maximal homomorphism. The kernel of any maximal homomorphism is a $Q$-ideal, then we have a quotient semiring and the fundamental theorem of homomorphism in semiring is applicable.

\section{REFERENCES}

P. J. Allen, A fundamental Theorem of homomorphism for semirings, Proc. Amer. Math. Soc., 21 (1969), 412-416.

Shahabaddin Ebrahimi Atani, The ideal theory in quotients of commutative semirings, Glasnik Matematicki (2007), 301308.

Indah P. Y., 2013, Teorema fundamental homomorfisma dan lokalisasi pada semiring, Skripsi, Yogyakarta, Jurusan Matematika Fakultas Matematika dan Ilmu Pengetahuan Alam Universitas Gajah Mada. 